\title{
Performance Characterization of High and Low Power Prism based Tunable Blue Laser Diodes Systems
}

\author{
Sani Mukhtar' ${ }^{1}$ J. A. Holguín-Lerma ${ }^{2}$, Islam Ashry ${ }^{2}$, Tien Khee $\mathrm{Ng}^{2}$, Boon S. Ooi ${ }^{2}$, and M. Z. M. Khan ${ }^{1, *}$ \\ ${ }^{1}$ Optoelectronics Research Laboratory, Electrical Engineering Department, King Fahd University of Petroleum and Minerals \\ (KFUPM), Dhahran 31261, Saudi Arabia \\ ${ }^{2}$ Computer, Electrical and Mathematical Sciences and Engineering (CEMSE) Division, King Abdullah University of Science \& \\ Technology (KAUST), Thuwal 23955-6900, Saudi Arabia \\ *zahedmk@kfupm.edu.sa
}

\begin{abstract}
Comparison of high- and low-power tunable external-cavity blue laser-diode system demonstrates a tunability of 10.6 and 4nm, respectively, with a corresponding SMSR as high as 35 and 32dB and linewidth as low as 97 and 59pm, while showcasing high stability at extreme operating conditions.
\end{abstract}

Keywords - Blue laser diode, self-injection locking, tunable lasers, external cavity diode lasers.

\section{INTRODUCTION}

The emerging deployment of blue semiconductor laser diodes (LDs) in different fields of applications, such as biomedical and spectroscopy, etc. [1] [2] necessitates improving their spectral purity and also the prospects of achieving a narrowband wavelength tunability. Wavelength selection mechanisms for achieving close to single longitudinal mode emission and wavelength tunability such as distributed feedback (DFB) gratings and external cavity configuration schemes (Littrow and Littman) are recently reported wherein a largest tuning range of $10.9 \mathrm{~nm}$ was achieved [3][4]. To possibly overcome the limitations of these reported approaches such as complexity, misalignment, cost, etc., a competing self-injection locking (SIL) scheme based external cavity diode laser (ECDL) system employing only a partial reflector was recently demonstrated on a green LD with a tuning range of $7.1 \mathrm{~nm}$ [5]. Moreover, very recently, we proposed a novel SL-ECDL system by integrating a prism and reported a record wavelength tuning range of $12 \mathrm{~nm}$ in blue region [6]. The system further demonstrated a side mode suppression ratio (SMSR) $>13 \mathrm{~dB}$, optical linewidth $(\Delta \lambda) \leq 110 \mathrm{pm}$ employing a high power Fabry Perot (FP) blue LD. However, the system stability at extreme operating conditions of high temperature and injection current, and the effect of deploying low power LD on the system performance has not been reported yet, which is crucial to substantiate the prospects of SIL-ECDL for practical deployment. Hence in this work, we present an in-depth performance analysis of the prism based SIL-ECDL system employing both the high power, $1.6 \mathrm{~W}$ (SN-LD-P06, SaNoor Technologies) and low power, $80 \mathrm{~mW}$ (PL450B, Thorlabs) commercial LDs. Comparing both the systems at similar operating conditions, the high-power system, demonstrate wide tunability of $10.6 \mathrm{~nm}$ with SMSR as high as $35 \mathrm{~dB}$ and $\Delta \lambda<120 \mathrm{pm}$, whereas a tunability of $4 \mathrm{~nm}$ with SMSR as high as $32 \mathrm{~dB}$ and $\Delta \lambda$ as low as $59 \mathrm{pm}$ was measured from the low power system, all at just above their threshold current and heat sink temperature of $30^{\circ} \mathrm{C}$. The stability analysis of the system under extreme operating condition showcases high performance and this substantiates its promising features for different applications.

\section{RESULTS AND DISCUSSION}

The SIL-ECDL system employing the two different LDs, viz. low-power and high-power follows the experimental set-up reported in Ref [6]. The results are plotted in Figs. 1(a) and 1(b), which show the wavelength tuning spectrum of the low- and high-power systems at just above the threshold current of 20 and $130 \mathrm{~mA}$, respectively, and at heat sink temperature of $30^{\circ} \mathrm{C}$, along the with corresponding extracted values of the SMSR and the linewidth. As seen from the figure, a tuning span of $3.7 \mathrm{~nm}(451.04-454.78 \mathrm{~nm})$, $\operatorname{SMSR}(\Delta \lambda)$ of $19 \pm 9 \mathrm{~dB}(89.5 \pm 30.5 \mathrm{pm})$ are measured from the low-power system. On the other front, the high-power system demonstrated a large tuning range of $10.6 \mathrm{~nm}(444.099-454.69 \mathrm{~nm})$, and SMSR $(\Delta \lambda)$ of $26.8 \pm 8.5 \mathrm{~dB}(113 \pm 7 \mathrm{pm})$. Moreover, Figs. 1 (c) and 1(d) summarize the performance of the low-power and high-power systems at different operating temperatures. As depicted from the figures, at room temperature of $20^{\circ} \mathrm{C}$ and at just above their threshold current, the low- and high-power systems respectively demonstrates a tuning window of $4 \mathrm{~nm}$ and $12 \mathrm{~nm}$ with corresponding SMSR $(\Delta \lambda)$ of $22.5 \pm 9.5 \mathrm{~dB}(75 \pm 15 \mathrm{pm})$ and $27.9 \pm 11.4$ $\mathrm{dB}(105 \pm 5 \mathrm{pm})$, respectively. Additionally, by operating the two systems at an extreme condition of three times their threshold currents and at $40^{\circ} \mathrm{C}$ temperature, performance degradation is apparent from both the systems, as depicted in Figs. 1(c) and (d) with shrinking wavelength tuning span, SMSR and $\Delta \lambda$ reaching average values of $\sim 18(\sim 17) \mathrm{dB}$ and $\sim 115(\sim 132)$ pm for the low- (high) power systems. In general, this performance degradation at harsh operating conditions is attributed to the dynamics of the external cavity system wherein large feedback power into the laser cavity translates to increase in various non-linear phenomena and modehopping, which makes precise locking difficult. However, it is worth mentioning at this instance that the low-power system 

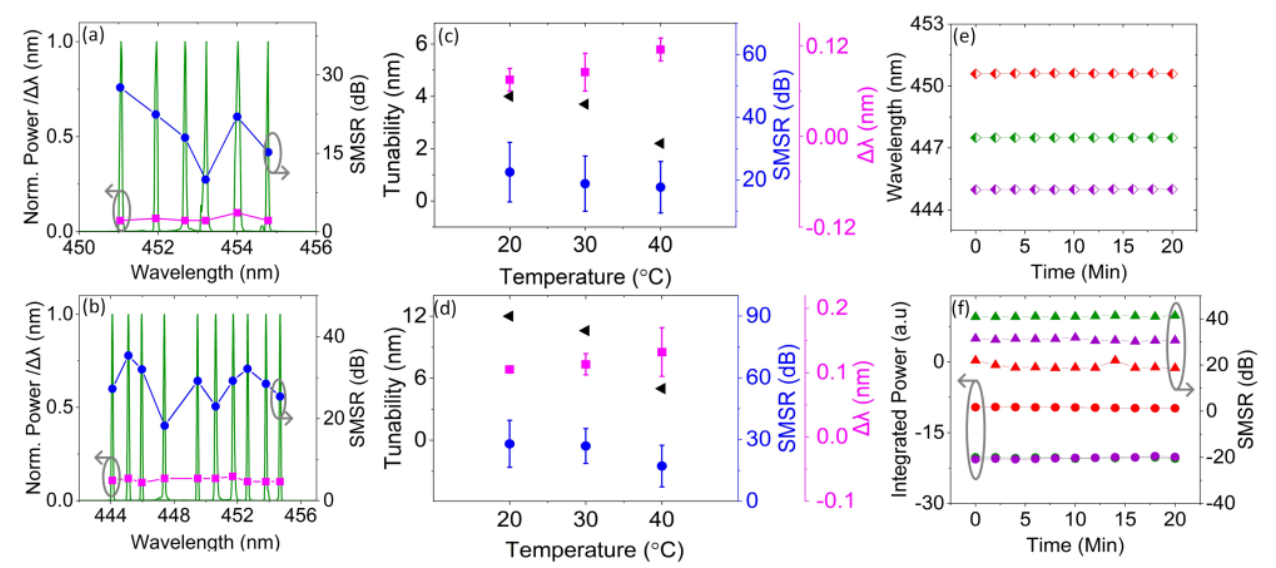

Fig. 1 Normalized lasing spectrums demonstrating the tuning window of (a) low-power and (b) high-power SIL-ECDL systems along with the corresponding extracted values of the SMSR and $\Delta \lambda$. Summary of the performance of (c) low-power and (d) high-power SIL-ECDL systems as a function of operating temperature, in terms of tunability (black inverted triangles), SMSR (blue closed circles), and linewidth (magenta closed squares). Performance of high-power SIL-ECDL system stability for three locked mode cases; $447.5 \mathrm{~nm}$ at $130 \mathrm{~mA}$ and $20^{\circ} \mathrm{C}$ (green), $444.97 \mathrm{~nm} 130 \mathrm{~mA}$ and $30^{\circ} \mathrm{C}$ (violet) and $450.60 \mathrm{~nm}$ at $390 \mathrm{~mA}$ and $40^{\circ} \mathrm{C}$ (red); in terms of (e) peak wavelength, (f) integrated power (circle) and the SMSR (triangles).

demonstrated narrower $\Delta \lambda$ with reasonable SMSR compared to the high-power system, but with reduced tunability. While the former observation could be associated to the relatively smaller amount of optical feedback power that assists in efficient injection locking, the latter one could be due to comparatively narrow gain profile of the low-power LD.

Furthermore, the high-power SIL-ECDL system stability is assessed for 20 minutes at time interval of 2 minutes and the extracted performance parameters in terms of the peak wavelength, integrated power and the SMSR are shown in Figs. 1(e) and (f). As depicted in Fig. 1(e), at just above threshold current of $130 \mathrm{~mA}$ and $20^{\circ} \mathrm{C}\left(30^{\circ} \mathrm{C}\right)$, the peak wavelength was extremely stable showing a negligible fluctuation of $447.505 \mathrm{~nm} \pm 5 \mathrm{pm}(444.98 \mathrm{~nm} \pm 11 \mathrm{pm})$ throughout the time span. Whereas the integrated power (SMSR) showed a minor fluctuation of $\pm 0.08 \mathrm{~dB}( \pm 0.3 \mathrm{~dB})$ and $\pm 0.15 \mathrm{~dB}( \pm 0.5 \mathrm{~dB})$ at $20^{\circ} \mathrm{C}\left(30^{\circ} \mathrm{C}\right)$. Moreover, increasing the injection current to $390 \mathrm{~mA}$ and the heat sink temperature to $40^{\circ} \mathrm{C}$, the high-power SIL-ECDL system was found to be very stable even at this extreme operating conditions. In this case, the peak wavelength exhibited a fluctuation of $450.605 \mathrm{~nm} \pm 15 \mathrm{pm}$ while the integrated power (SMSR) demonstrated a variation of $\pm 0.42 \mathrm{~dB}( \pm 1.65 \mathrm{~dB})$. This stable features of the system at harsh operating conditions reinstate the robustness of the proposed system and strengthens its promise for practical applications.

\section{CONCLUSION}

In summary, the performance characteristics of low- and high-power prism based tunable self-injection locked external cavity blue laser diode system are investigated and compared. The high-power system exhibited a wideband tuning range of $10.6 \mathrm{~nm}$ with SMSR as high as $35 \mathrm{~dB}$, and linewidth $<120 \mathrm{pm}$, but the low power LD exhibited tuning range of $4 \mathrm{~nm}$, SMSR as high as $32 \mathrm{~dB}$ with linewidth $<100 \mathrm{pm}$. The stability evaluation of the system under extreme operating conditions further substantiate the promising features of the prism based tunable SIL system for different fields of applications.

\section{ACKNOWLEDGMENT}

The authors (SM and MZMK) thank King Fahd University of Petroleum and Minerals (KFUPM) for supporting this research through King Abdulaziz City for Science and Technology (KACST), Technology Innovation Center (TIC) for Solid-State Lighting sub-awarded grant EE002381, and primary grant KACST TIC R2-FP- 008. The authors (JAHL, IA, TKN and BSO) acknowledge the support from King Abdullah University of Science and Technology (KAUST) through baseline funding BAS/1/1614-01-01 and KAUST-KFUPM special Initiative (KKI) program (REP/1/2878-01-01).

\section{REFERENCES}

[1] A. Müller et al., "Diode laser based light sources for biomedical applications," Laser Photonics Rev., vol. 7, no. 5, pp. 605-627, 2013.

[2] I. S. Burns, J. Hult, and C. F. Kaminski, "Spectroscopic use of a novel blue diode laser in a wavelength region around $450 \mathrm{~nm}$," Appl. Phys. B Lasers Opt., vol. 79, no. 4, pp. 491-495, 2004.

[3] J. A. Holguin-Lerma, M. Kong, O. Alkhazragi, X. Sun, T. Khee Ng, and B. S. Ooi, "480-nm distributed-feedback InGaN laser diode for 105-Gbit/s visible-light communication," Opt. Lett., vol. 45, no. 3, p. 742, 2020.

[4] D. Ding, X. Lv, X. Chen, F. Wang, J. Zhang, and K. Che, “Tunable high-power blue external cavity semiconductor laser," Opt. Laser Technol., vol. 94, pp. 1$5,2017$.

[5] M. H. M. Shamim, T. K. Ng, B. S. Ooi, and M. Z. M. Khan, “Tunable self-injection locked green laser diode,” Opt. Lett., vol. 43, no. 20, p. 4931, 2018.

[6] S. Mukhtar, I. Ashry, C. Shen, T. K. Ng, B. S. Ooi, and M. Z. M. Khan, "Blue Laser Diode System With an Enhanced Wavelength Tuning Range," IEEE Photonics J., vol. 12, no. 2, pp. 1-10, 2020. 